\title{
On EPR-type Entanglement in the Experiments of Scully et Al. I. The Micromaser Case and Delayed-Choice Quantum Erasure
}

\section{F. Herbut}

\begin{abstract}
Delayed-choice erasure is investigated in two-photon two-slit experiments that are generalizations of the micromaser experiment of Scully et al. [Scully, M. O. et al. Nature 351, 111-116 (1991)]. Applying quantum mechanics to the localization detector, it is shown that erasure with delayed choice in the sense of Scully, has an analogous structure as simple erasure. The description goes beyond probabilities. The EPR-type disentanglement, consisting in two mutually incompatible distant measurements, is used as a general framework in both parts of this study. Two simple coherence cases are shown to emerge naturally, and they are precisely the two experiments of Scully et al. The treatment seems to require the relative-reality-of-unitarilyevolving-state (RRUES) approach . Besides insight in the experiments, this study has also the goal of insight in quantum mechanics. The question is if the latter can be more than just a "book-keeping device" for calculating probabilities as Scully et al. modestly and cautiously claim.
\end{abstract}

Keywords Delayed-choice erasure. Distant measurement. EPR-type disentanglement. Detector as quantum-mechanical system. Relative-reality-ofunitarily-evolving-state approach. Relative-state interpretation.

\section{Introduction}

The fascinating process of quantum erasure (of entanglement), even its more sophisticated delayed-choice version, were first discussed by Edwin Jaynes [1], but, as far as I can tell, without giving any terms for the general phenomenon.

F. Herbut (mail)

Serbian Academy of Sciences and Arts, Knez Mihajlova 35, 11000 Belgrade, Serbia

e-mail: fedorh@infosky.net and from USA etc. fedorh@mi.sanu.ac.yu 
Marlan O. Scully and coworkers elaborated it [2], [3].

Particularly baffling is the delayed-choice (or after-detection) version of erasure in contrast to the simple(or before-detection) case. (One should distinguish the mentioned delayed choice in the sense of Scully from that in the sense of Wheeler [4.)

One of the first attempts to perform a real erasure experiment [5] presented its theoretical part in second quantization. However, it was demonstrated [6], along the same lines as in this study, that first-quantization quantum-mechanical insight is feasible and useful. But, for consistency, this line of approach seems to require the relative-reality-of-unitarily-evolvingstate or, shortly the RRUES, interpretation, which will be explained in this article.

The claim of Scully and Walther [7] that there is no essential difference between simple and delayed-choice erasures is further elaborated in this article using the reality-of-states approach. The slight difference that does exist will also be discussed.

One should clarify, for the reader's benefit, that a bipartite state is correlated whenever it is not factorizable (tensorically) into the states of its subsystems. Not all correlations are entanglement. Separable mixed states, i. e., ones that are mixtures of uncorrelated states, are correlated (unless they have only one term), but have no entanglement. Contrariwise, the correlations in any bipartite pure state consist only of entanglement.

The notion of an EPR-type bipartite pure state, or, equivalently one that contains EPR-type entanglement, also requires clarification. By definition, $|\Phi\rangle_{I, I I}$ is an EPR-type bipartite state vector if one can have EPR-type disentanglement, i. e., if one can perform distant (direct-interaction-free) measurement (cf [8]) of either of two mutually incompatible subsystem observables.

The notion of disentanglement (as well as of entanglement) goes back to Schrödinger [9].

\section{Does Interaction Destroy Coherence?}

The authors remark in their thorough analysis of erasure [7] (p. 399):

"Just how the acquisition of which way (Welcher Weg) information 
rubs out the interference fringe is an interesting question."

As a contrast to their answer, they quote Feynman, who comments on Young's two-slit interference case [10], [11]:

"If an apparatus is capable of determining which hole the electron (or atom or ...) goes through, it cannot be so delicate that it does not disturb the pattern in an essential way."

The answer of the authors then goes as follows ([7], p. 400).

"... it is not necessarily the indelicate nature of our probing that rubs out the interference pattern. It is simply knowing (or having the ability to know even if we choose not to look at the Welcher Weg detector) which eliminates the pattern. This has been verified experimentally."

I agree, but I'd like to approach the matter differently to gain additional insight.

Let us take a simple case of coherence like e. g.

$$
\begin{gathered}
|\phi\rangle_{I I} \equiv \alpha|1\rangle_{I I}+\beta|2\rangle_{I I}, \\
\left\langle\left. i\right|_{I I} \mid j\right\rangle_{I I}=\delta_{i j}, \quad i, j=1,2 ; \quad|\alpha|^{2}+|\beta|^{2}=1, \quad \alpha \neq 0 \neq \beta .
\end{gathered}
$$

The state vectors $|j\rangle_{I I}, j=1,2$, may, e. g., describe transition of the slits in the Young experiment. Then $|\phi\rangle_{I I}$ of (1a) is the coherent state that gives fringes on the screen with detectors.

If another system $I$ has been interacting with the system described by $|\phi\rangle_{I I}$ in the way of ideal measurement, or some similar process has taken place, which has led to a bipartite state vector

$$
|\Phi\rangle_{I, I I}=\alpha^{\prime}|1\rangle_{I}|1\rangle_{I I}+\beta^{\prime}|2\rangle_{I}|2\rangle_{I I}, \quad\left|\alpha^{\prime}\right|^{2}+\left|\beta^{\prime}\right|^{2}=1, \quad \alpha^{\prime} \neq 0 \neq \beta^{\prime}
$$

with $\left\langle\left. i\right|_{I} \mid j\right\rangle_{I}=\delta_{i j}, \quad i, j=1,2$, then the subsystem state (reduced density operator) $\rho_{I I} \equiv \operatorname{tr}_{I}\left(|\Phi\rangle_{I, I I}\left\langle\left.\Phi\right|_{I, I I}\right) \quad\right.$ of subsystem $I I$ is

$$
\rho_{I I}=\left|\alpha^{\prime}\right|^{2}|1\rangle_{I I}\left\langle\left. 1\right|_{I I}+\left|\beta^{\prime}\right|^{2} \mid 2\right\rangle_{I I}\left\langle\left. 2\right|_{I I} .\right.
$$

Comparing (1a) and (3), we see that coherence has been eliminated in subsystem II, but it is not destroyed. We see in (2) that coherence reappears in the state of the composite system. Coherence being elevated to the 
larger system by interaction of the parts or by some analogous process is a remarkable fact in quantum mechanics (cf [12]). It is an obvious consequence of the linear nature of the unitary evolution operator of the composite system (which includes the interaction etc.).

Coherence in $|\Phi\rangle_{I, I I}$ of (2) implies entanglement between the subsystems.

\section{Simplest EPR-type Entanglement}

Let us assume that a bipartite state vector $|\Psi\rangle_{I, I I}$ is given. It can always be written in the form of a so-called canonical Schmidt decomposition, which is in terms of bi-orthonormal bases with positive expansion coefficients. (For a concise review see Subsection 2.1 in [13.)

To treat the simplest case, we assume that the so-called Schmidt rank, i. e., the number of terms in the decomposition (which is an invariant with respect to the different biorthogonal decompositions) is two:

$$
|\Psi\rangle_{I, I I}=r_{1}^{1 / 2}|1\rangle_{I}|1\rangle_{I I}+r_{2}^{1 / 2}|2\rangle_{I}|2\rangle_{I I} .
$$

A practical advantage of the canonical Schmidt decomposition is that one can read in it the spectral forms of the subsystem states (reduced density operators):

$$
\begin{gathered}
\rho_{I} \equiv \operatorname{tr}_{I I}\left(|\Psi\rangle_{I, I I}\left\langle\left.\Psi\right|_{I, I I}\right)=r_{1}|1\rangle_{I}\left\langle\left. 1\right|_{I}+r_{2} \mid 2\right\rangle_{I}\left\langle\left. 2\right|_{I},\right.\right. \\
\rho_{I I}=r_{1}|1\rangle_{I I}\left\langle\left. 1\right|_{I I}+r_{2} \mid 2\right\rangle_{I I}\left\langle\left. 2\right|_{I I} .\right.
\end{gathered}
$$

Physically, a canonical Schmidt decomposition, like (4), plays an important role in distant measurement. If one measures (in a predictive way) the observable consisting in determining if the subsystem $I$ is in the state $|1\rangle_{I}$ or $|2\rangle_{I}$, then one has the change of state from (4) to $|j\rangle_{I}|j\rangle_{I I}, j=1$ or 2 , which implies that the (technically) 'distant' subsystem $I I$ is brought into the state $|j\rangle_{I I}, j=1,2$. ('Distantness' consists in lack of interaction between subsystems $I$ and $I I$.) Distant measurement takes place without interaction of the measuring apparatus with the distantly measured subsystem ( $I I$ in our example). It is a consequence of the direct measurement on subsystem $I$ and of the quantum correlations between subsystems $I$ and $I I$. 
The very possibility of the described distant measurement is the whichway knowledge that subsystem $I$ has about subsystem II (and vice versa). If we do perform the measurement, then also we acquire this knowledge.

In general, one has EPR-type entanglement if there is degeneracy in at least one of the positive eigenvalues of the reduced density operators [14. (For EPR-type entanglement see also [15]). In (4) this means $r_{1}=r_{2}$. Hence, (4) and (5b) become

$$
|\Psi\rangle_{I, I I}=(1 / 2)^{1 / 2}\left(|1\rangle_{I}|1\rangle_{I I}+|2\rangle_{I}|2\rangle_{I I}\right) .
$$

and

$$
\rho_{I I}=(1 / 2)|1\rangle_{I I}\left\langle\left. 1\right|_{I I}+(1 / 2) \mid 2\right\rangle_{I I}\left\langle\left. 2\right|_{I I}\right.
$$

respectively.

Now the canonical Schmidt decomposition has a non-denumerably infinite degeneracy, viz., every orthonormal basis in the two-dimensional range of $\rho_{I}$ is part of an an eigen-basis of $\rho_{I}$ (and symmetrically for $\rho_{I I}$ ), and (generalized) expansion in it leads to a canonical Schmidt decomposition (cf Subsection 2.1 in [13]).

To be concrete, let

$$
|a\rangle_{I} \equiv e^{i \lambda} p|1\rangle_{I}+e^{i \delta} q|2\rangle_{I}, \quad 0<p, q<1, \quad p^{2}+q^{2}=1, \quad 0 \leq \lambda, \delta<2 \pi
$$

be an arbitrary coherence state vector (a linear combination with both coefficients non-zero) in the range of $\rho_{I}$. Then, as easily checked, the obviously unique (up to a phase factor) orthogonal state vector has the form

$$
|b\rangle_{I} \equiv e^{i \gamma} q|1\rangle_{I}+e^{i(\gamma+\delta-\lambda+\pi)} p|2\rangle_{I}, \quad 0 \leq \gamma<2 \pi .
$$

To expand the state vector $|\Psi\rangle_{I, I I} \quad(\mathrm{cf}(6 \mathrm{a}))$ in the sub-basis $\left\{|a\rangle_{I}\right.$, $\left.|b\rangle_{I}\right\}$, we define

$\overline{|a\rangle}_{I I} \equiv e^{-i \lambda} p|1\rangle_{I I}+e^{-i \delta} q|2\rangle_{I I}, \quad \overline{|b\rangle}_{I I} \equiv e^{-i \gamma} q|1\rangle_{I I}+e^{-i(\gamma+\delta-\lambda+\pi)} p|2\rangle_{I I}$. $(8 a, b)$

Then, as shown in Appendix A, the same bipartite state vector (6a) has the alternative canonical Schmidt decomposition

$$
|\Psi\rangle_{I, I I}=(1 / 2)^{1 / 2}\left(|a\rangle_{I} \overline{|a\rangle}_{I I}+|b\rangle_{I} \overline{|b\rangle}_{I I}\right) .
$$


The physical meaning of (9) is analogous to that of (6a) (or (4)) explained above: If one performs a direct measurement on subsystem $I$ to find out if it is in the state $|a\rangle_{I}$ or $|b\rangle_{I}$, ipso facto one finds subsystem $I I$ (by distant measurement) in the corresponding, i. e., 'partner', state $\overline{|a\rangle}_{I I}$ or $\overline{|b\rangle}_{I I}$ respectively.

The possibility to perform, in principle, either of the two mutually incompatible distant measurements based on (6a) or (9) respectively, is called EPR-type disentanglement. (The original EPR paper [16] dealt with EPRtype disentanglement of position and linear momentum.) A case of EPR-type disentanglement performed in one real experiment will be discussed in Part II of this study.

Distant measurement based on the basic canonical Schmidt decomposition (6a) is often feasible. In all versions of the Young two-slit experiment with pairs of particles it is called 'which-way' measurement.

Concerning linearly polarized photons, when in $|j\rangle \quad j$ refers to two mutually orthogonal linear polarizations, experiments that establish the EPRtype entanglement in (6a) have been performed. (See the $J=0 \rightarrow J=$ $1 \rightarrow J=0$ atomic cascade transitions as source of photon pairs - $J$ being the atomic angular momentum [17.)

If in $|j\rangle \quad j$ in (6a) means spin-up and spin-down of a spin-one-half particle, then (6a) gives one of the vectors in the so-called Bell operator basis used in the discovery of teleportation [18]. Distant spin-projection measurement is here certainly feasible.

Thus, EPR-type correlations have been studied in great detail theoretically and experimentally, but, as far as I know, without actual EPR-type disentanglement experiments.

Performing distant measurement based on the canonical Schmidt decomposition (9) requires the possibility to measure the basis $(7 \mathrm{a}, \mathrm{b})$ in direct measurement. This takes exceptional experimental skill.

It will be shown that the experiments of Scully et al. that appeared in the literature are restricted to simple coherence bases, the first one:

$$
| \pm\rangle_{I} \equiv(1 / 2)^{1 / 2}\left(|1\rangle_{I} \pm|2\rangle_{I}\right)
$$

and the second one

$$
| \pm i\rangle_{I} \equiv(1 / 2)^{1 / 2}\left(|1\rangle_{I} \pm i|2\rangle_{I}\right)
$$

(It is obvious from (7a) and (7b) that (10) and also (11) are orthogonal sets.) 
These bases are not just mathematically simple. They have simple physical properties with respect to $|\Psi\rangle_{I, I I}$ (cf (6a)). Namely, the first simple coherence basis $| \pm\rangle_{I}$, which is given by (10), is the only coherence basis determining, via expansion of $|\Psi\rangle_{I, I I}$, a canonical Schmidt decomposition in which each term is symmetric under the (two-particle) exchange operator (assuming $p=q$ in (7a) for simplicity):

$$
\begin{aligned}
|\Psi\rangle_{I, I I}= & (1 / 2)^{1 / 2}\left\{\left[(1 / 2)^{1 / 2}\left(|1\rangle_{I}+|2\rangle_{I}\right)\right]\left[(1 / 2)^{1 / 2}\left(|1\rangle_{I I}+|2\rangle_{I I}\right)\right]+\right. \\
& {\left.\left[(1 / 2)^{1 / 2}\left(|1\rangle_{I}-|2\rangle_{I}\right)\right]\left[(1 / 2)^{1 / 2}\left(|1\rangle_{I I}-|2\rangle_{I I}\right)\right]\right\} }
\end{aligned}
$$

(cf (9) with $(8 \mathrm{a}, \mathrm{b}))$. This property is physical due to the distant-measurement interpretation of the decomposition (explained above).

The second simple coherence basis $| \pm i\rangle_{I} \quad(\mathrm{cf}(11))$ is determined as being the only coherence basis in the corresponding canonical Schmidt decomposition of which the exchange operator maps the first term on the rhs onto the second one and vice versa:

$$
\begin{aligned}
|\Psi\rangle_{I, I I}= & (1 / 2)^{1 / 2}\left\{\left[(1 / 2)^{1 / 2}\left(|1\rangle_{I}+i|2\rangle_{I}\right)\right]\left[(1 / 2)^{1 / 2}\left(|1\rangle_{I I}-i|2\rangle_{I I}\right)\right]+\right. \\
& {\left.\left[(1 / 2)^{1 / 2}\left(|1\rangle_{I}-i|2\rangle_{I}\right)\right]\left[(1 / 2)^{1 / 2}\left(|1\rangle_{I I}+i|2\rangle_{I I}\right)\right]\right\} }
\end{aligned}
$$

These claims are proved in Appendix B.

The coherence canonical Schmidt decompositions (12) and (13) obviously imply the following decompositions of the state of subsystem II, which consists of coherence terms:

$$
\begin{gathered}
\rho_{I I}=(1 / 2)\left\{[ ( 1 / 2 ) ^ { 1 / 2 } ( | 1 \rangle _ { I I } + | 2 \rangle _ { I I } ) ] \left[( 1 / 2 ) ^ { 1 / 2 } \left(\left\langle\left. 1\right|_{I I}+\left\langle\left. 2\right|_{I I}\right)\right]+\right.\right.\right. \\
{\left[(1 / 2)^{1 / 2}\left(|1\rangle_{I I}-|2\rangle_{I I}\right)\right]\left[(1 / 2)^{1 / 2}\left(\left\langle\left. 1\right|_{I I}-\left\langle\left. 2\right|_{I I}\right)\right]\right\},\right.}
\end{gathered}
$$

and

$$
\begin{gathered}
\rho_{I I}=(1 / 2)\left\{[ ( 1 / 2 ) ^ { 1 / 2 } ( | 1 \rangle _ { I I } + i | 2 \rangle _ { I I } ) ] \left[( 1 / 2 ) ^ { 1 / 2 } \left(\left\langle\left. 1\right|_{I I}-i\left\langle\left. 2\right|_{I I}\right)\right]+\right.\right.\right. \\
{\left[(1 / 2)^{1 / 2}\left(|1\rangle_{I I}-i|2\rangle_{I I}\right)\right]\left[(1 / 2)^{1 / 2}\left(\left\langle\left. 1\right|_{I I}+i\left\langle\left. 2\right|_{I I}\right)\right]\right\} .\right.}
\end{gathered}
$$


(Note that also (6b) is valid).

The well-known micromaser thought experiment of Scully et al. [3] was based on the simple coherence basis (10) and the corresponding canonical Schmidt decomposition (12). In outline the experiment goes as follows.

A plane atom wave split into two coherent collimated beams enters microwave cavities. Its passage through the cavities forces the previously excited atom to emit a photon (subsystem $I$ ). As long as the shutters remain closed, the photon is found inside one of the cavities, and which-way information is obtained, i. e., $|1\rangle_{I}\left\langle\left. 1\right|_{I} \text { or } \mid 2\right\rangle_{I}\left\langle\left. 2\right|_{I}\right.$ is detected. No interference is observed on the atoms (subsystem II, cf the two terms on the rhs of (6b)).

When the shutters are opened, the which-way information is erased: Atoms associated with the photons that are subsequently detected by a photo-sensor give rise to the same interference pattern that would be observed without the cavities ( $\mathrm{cf}(1 \mathrm{a})$ with $\alpha=\beta=(1 / 2)^{1 / 2}$ ) because it is the symmetric field $(1 / 2)^{1 / 2}\left(|1\rangle_{I}+|2\rangle_{I}\right)$ that is actually detected. The remaining atoms (subsystems $I I$ in the state $(1 / 2)^{1 / 2}\left(|1\rangle_{I I}-|2\rangle_{I I}\right)$ ) correspond to the antisymmetric field, and they give rise to the complementary interference pattern.

This is a beautiful illustration of EPR-type disentanglement because both mutually incompatible distant measurements are seen to be performable (at least in principle).

The remarkable real random-choice and delayed-choice erasure experiment of Kim et al. [19] was based on the second simple coherence basis (11) and the corresponding canonical Schmidt decomposition (13). This will be proved in the second part of this study.

It should be noted that coherence is a relative notion: The state vectors $|+\rangle_{I I}$ and $|-\rangle_{I I}$ are coherent with respect to the state vectors $|1\rangle_{I I}$ and $|2\rangle_{I I}$, which are assumed to have basic physical meaning in the experiment discussed (e. g., passing the slits 1 and 2 respectively). Mathematically, also the reverse relation is true: the latter two state vectors are coherent with respect to the former two. But this is physically irrelevant in the case at issue.

If the interaction described in the preceding section has rubbed out interference, then the coherence distant measurement based on (10) and (12) 
reestablishes coherence in subsystem $I I$. This is erasure (of entanglement). If direct measurement of the states $| \pm\rangle_{I}$ is performed before or after localization measurement in the state $\rho_{I I}$, we have simple erasure (beforedetection erasure) or delayed-choice erasure (after-detection erasure) respectively.

It will be shown that both types of erasure are EPR-type disentanglement with the only difference that in simple erasure no detector has to be included in the quantum-mechanical description, whereas in delayed-choice erasure, to 'see' the EPR-type disentanglement, quantum mechanics has to be applied also to the localization detectors for particle $I I$.

We are now going to prepare the ground for treatment of delayed-choice erasure.

\section{Subsystem Interaction}

Let us assume that subsystem $I I$ interacts with a third system $D_{I I}$ (we have primarily a detector in mind) in some state that we denote by $|0\rangle_{D_{I I}}$ while the former is a subsystem of the composite system in the state $|\Psi\rangle_{I, I I}$ ( $\mathrm{cf}(6 \mathrm{a})$ or (9)). Let us describe the interaction by a unitary evolution operator $U_{I I D_{I I}}$. Also evolution for some time after the interaction has ceased may be included. For simplicity, we disregard the possible changes caused by evolution of subsystem $I$.

The complementary (which-way and coherence) decompositions (6a) and (9) lead to

$$
\begin{gathered}
\left(1_{I} \otimes U_{I I D_{I I}}\right)\left(|\Psi\rangle_{I, I I}|0\rangle_{D_{I I}}\right)= \\
(1 / 2)^{1 / 2}|1\rangle_{I} \otimes\left[U_{I I D_{I I}}\left(|1\rangle_{I I}|0\rangle_{D_{I I}}\right)\right]+ \\
(1 / 2)^{1 / 2}|2\rangle_{I} \otimes\left[U_{I I D_{I I}}\left(|2\rangle_{I I}|0\rangle_{D_{I I}}\right)\right]
\end{gathered}
$$

and

$$
\begin{aligned}
& \left(1_{I} \otimes U_{I I D_{I I}}\right)\left(|\Psi\rangle_{I, I I}|0\rangle_{D_{I I}}\right)= \\
& (1 / 2)^{1 / 2}|a\rangle_{I} \otimes\left[U_{I I D_{I I}}\left(\overline{|a\rangle}_{I I}|0\rangle_{D_{I I}}\right)\right]+ \\
& (1 / 2)^{1 / 2}|b\rangle_{I} \otimes\left[U_{I I D_{I I}}\left(\overline{|b\rangle}_{I I}|0\rangle_{D_{I I}}\right)\right]
\end{aligned}
$$

respectively. 
Since $|1\rangle_{I I}$ and $|2\rangle_{I I}$ are orthogonal, so are $\left(|1\rangle_{I I}|0\rangle_{D_{I I}}\right)$ and $\left(|2\rangle_{b}|0\rangle_{D_{I I}}\right)$, and, on account of the unitary nature of the evolution operator, so are also $U_{I I D_{I I}}\left(|1\rangle_{I I}|0\rangle_{D_{I I}}\right)$ and $U_{I I D_{I I}}\left(|2\rangle_{I I}|0\rangle_{D_{I I}}\right)$. The analogous argument holds for evolution (17). Hence, (16) and (17) are still two complementary canonical Schmidt decompositions of a bipartite $I+I I D_{I I}$ EPR-type state vector. Viewing the lhs of these relations as a tripartite state, clearly the state of the subsystem $I+I I$ does no longer have the nice EPR-type coherence of (6a) or (9). (The change depends on the structure of the evolution operator).

To simplify the exposition, we now confine the general coherence basis $\left\{|a\rangle_{I},|b\rangle_{I}\right\}$ (cf (7a) and (7b)) to the two simple choices (cf (10) and (11)), which are the only relevant ones in this study. (However, the argument is valid for the general case.)

Further, one can make use of the linearity of the evolution operator. Then substitution of (10) in (17) gives

$$
\begin{gathered}
U_{I I D_{I I}}\left(\overline{| \pm\rangle}_{I I}|0\rangle_{D_{I I}}\right)=(1 / 2)^{1 / 2} U_{I I D_{I I}}\left(|1\rangle_{I I}|0\rangle_{D_{I I}}\right) \pm \\
(1 / 2)^{1 / 2} U_{I I D_{I I}}\left(|2\rangle_{I I}|0\rangle_{D_{I I}}\right) .
\end{gathered}
$$

An analogous argument is valid for substitution of the second simple coherence basis $| \pm i\rangle \quad(\mathrm{cf}(11))$ in (17).

Taking the concrete case of Young's two-slit interference in some version, one can view $U_{I I D_{I I}}\left(|j\rangle_{I I}|0\rangle_{D_{I I}}\right), j=1,2$, as describing the sub-ensemble of atoms (photons etc.) that have passed slit $j$ and have interacted afterwards. Then these are still the definite-way states without coherence. Contrariwise, the states $U_{I I D_{I I}}\left(\overline{| \pm\rangle}_{I I}|0\rangle_{D_{I I}}\right)$, and the states $U_{I I D_{I I}}\left(\overline{| \pm i\rangle}_{I I}|0\rangle_{D_{I I}}\right)$ are coherence states as clear from (18).

It is very important to note that system $D_{I I}$ is, in principle, any system (that can interact with subsystem II. ) Further, we are dealing with any interaction.

One should also note that one has a composite EPR-type state vector of the bipartite system $D_{I} I+I I D_{I I}$ also if any system $D_{I}$ interacts in any way with subsystem $I$. Namely, any unitary evolution operator $U_{D_{I} I} \otimes U_{I I D_{I I}}$ does not change the form of the canonical Schmidt dacomposition of the composite state as long as there is no inteaction between subsystems $D_{I} I$ and $I I D_{I I}$. (For more details, see Appendix C.) 


\section{Delayed-choice Erasure}

Now we make the often done, but controversial, assumption that also macroscopic systems, in particular detectors, which are usually described by classical physics, can, in principle, be treated by the quantum-mechanical formalism, and that they can be in pure states.

Let system $D_{I I}$ in the preceding section be the detector (or system of detectors) measuring the localization of particle $I I$. Then, as it was argued

above, the states $U_{I I D_{I I}}\left(\overline{| \pm\rangle}_{I I}|0\rangle_{D_{I I}}\right)$ or $U_{I I D_{I I}}\left(\overline{| \pm i\rangle}_{I I}|0\rangle_{D_{I I}}\right) \quad(\mathrm{cf}(10)$ and (11)) are obtained in delayed-choice erasure, i. e., if the detection takes place before the direct measurement on subsystem $I$ ascertaining if it is in the state $|+\rangle_{I}$ or $|-\rangle_{I}$ is performed (analogously for the second simple coherence basis).

It was demonstrated [20] (Subsection III.A) that, as far as the relevant probabilities are concerned, there is no difference between simple and delayedchoice erasure. The straightforward demonstration of the authors is directly applicable to the more general case treated in this article because it is due, as they point out, to the mathematical properties of the tensor product.

To enable the reader to obtain a fuller appreciation of the approach of this paper, a treatment of delayed-choice erasure that implies the claimed equality of the relevant probabilities is presented in Appendix D.

The EPR-type entanglement discussed in this article is mutatis mutandis applicable also to the mentioned real experiment reported by Kim, Yu, Kulik, Shih, and Scully [19]. It contains an additional upgrading of delayed-choice erasure: it is no longer the experimenter, but an automatic random choice that determines if which-way or coherence distant measurement is taking place. It can also be viewed as an EPR-type disentanglement in terms of two mutually incompatible distant measurements. This is explained in the second part of the present study.

\section{Summing Up}

We now sum up the conceptual steps involved in delayed-choice erasure viewed as EPR-type disentanglement.

(i) Some mechanism rubs out the coherence in system II (cf (1a)) 
elevating it it to the larger system $I+I I \quad(\mathrm{cf}(2))$. This implies entanglement between the subsystems enabling e. g. subsystem $I$ to 'mark' in terms of its states $|q\rangle_{I}, q=1,2$, the corresponding states of subsystem $I I$ (cf (2)). This is a kind of 'knowledge' on part of subsystem $I$ about subsystem $I I$.

(ii) Performing direct measurement on subsystem $I$ in the bipartite state (6a) to learn in which of the states $|j\rangle_{I}, j=1,2$, it is found, one, by this very act, performs distant measurement on subsystem II finding out if it is in the state $|1\rangle_{I I}$ or $|2\rangle_{I I}$. (For a more general discussion of distant measurement see [8].) In this way the experimenter 'shares' the mentioned 'knowledge' of subsystem $I$ about subsystem $I I$.

(iii) In step (i) one actually arrives at a coherent bipartite state vector (2) with $|\alpha|=|\beta|$. This gives EPR-type entanglement, which allows distant measurement of incompatible observables. (This is what we call EPR-type disentanglement.) In particular, the composite state $|\Psi\rangle_{I, I I}$ allows also distant measurement of the states $|j\rangle_{I I}, j= \pm$ (cf (12)).

(iv) Any interaction of subsystem $I I$ with any other system $D_{I I}$ leads to EPR-type composite-system states (and EPR-type entanglement between $I$ and $\left.I I D_{I I}\right)$. One has, e. g., the canonical Schmidt decompositions (16) and (17), which allow incompatible distant measurements: the which-way one and the which-coherence

one.

(v) System $D_{I I}$ can be the detector for particle $I I$ in delayed-choice erasure, and the interaction can be that in the corresponding localization measurement.

\section{Concluding Remarks}

A) The assumption that one can, in principle, describe the macroscopic detector by quantum mechanics, like any microscopic system, is controversial. Let me give two facts supporting the assumption.

(i) Niels Bohr himself felt occasionally the need to describe macroscopic bodies by quantum mechanics. To illustrate this, I'll quote his words ([21], p. 50):

... heavy bodies like diaphragms and shutters... . ..., in contrast to the proper measuring instruments, these bodies together with the par- 
ticles would constitute the system to which the quantum-mechanical formalism has to be applied." (Italics by F. H.)

The same was quoted by Shimony [22]. He commented upon it as follows (p. 770):

"Bohr is saying that from one point of view the apparatus is described classically and from another, mutually exclusive point of view, it is described quantum mechanically."

(ii) As it is well known, there exist quantum systems that are macroscopic like e. g. superconductors, quantum fluids etc. There is a tendency to prove experimentally that quantum mechanics is valid, in principle, for all macroscopic objects. Important progress was achieved along these lines (see e. g. [23]). The validity of quantum mechanics, in particular, of the superposition principle, was demonstrated, but only for mesoscopic systems so far (as far as known to the author).

B) An argument against treating detectors (and other macroscopic objects) in the same way as microscopic ones, as done in this article, constitutes the fact that the former are constantly and strongly dynamically and statistically coupled to the environment. Modern decoherence theory [24], 25], 26] has established this beyond doubt. The obvious way to circumvent the objection is to join the environment (or at least part of it) to the detector etc. as if it all were one quantum system. (The point to note is that the experimenter has to be in the 'subject' of quantum-mechanical description.)

C) Bohr's words quoted in A(i) imply acceptance of displacement of the so-called cut of Heisenberg (cut between object and observer-system or 'object' and 'subject' as it is often said). The physics does not change in such displacement. This has been established in the famous psycho-physical parallelism of von Neumann [27] (p. 420).

Bohr's acceptance of displacement of the cut can be viewed as a smooth connection between the Copenhagen interpretation [28] and that of Everett [29] if the latter is understood as saying that the object of quantum-mechanical description is relative to the preparator (and, possibly, to part of its state) and to the measuring instrument (and, possibly, to part of its state, primarily one displaying the result) when one applies quantum mechanics to an experiment. An analysis [30] of Mott's famous quantum-mechanical insight in the 
formation of droplet-trajectories in bubble chambers led to relative-collapse ideas (a year earlier than Rovelli's noted similar ideas [31] that he called 'relational').

Let me point out that von Neumann's mentioned theorem on psychophysical parallelism implies that if one and the same chain of subsystems is part of the 'object' in two different cuts, the chain has the same physical description. (I do not mean just probabilities; I mean the reality-of-state approach, which contains the probabilities.)

To be concrete, the bipartite $I+I I$ system in the case of simple erasure treated in this article (an example of the chain) is equally described in the version when the cut is such that the detector $D_{I I}$ of $I I$ is part of the subject and in the version when it is part of the object. This is presented in more detail in Appendix $\mathrm{C}$.

The authors of Ref. [20] claim (p. 328, left column): "It appears that Mohrhoff is led astray by regarding the state reductions ... as physical processes, rather than accepting that they are nothing but mental processes." (See more about the controversy itself in remark $\mathrm{H}$ below.)

In the reality-of-state approach adopted in this article one avoids attributing objectivity to collapse by utilizing a kind of Everett-like relative state approach as stated above. The "mental process" is then the choice of the cut, and what belongs to the 'object' and what to the 'subject'. One actually chooses in this way what aspect of the quantum reality one wants to 'highlight', i. e., make part of the object.

It turns out that the quantum formalism can provide us only with relative reality: with respect to the preparator (and, possibly, part of its state) and regarding our choice of the 'subject'. In a quantum-mechanical analysis of an experiment, the experimenter is necessarily part of the subject; he is even the source of the decision where the cut should be. Unfortunately, this is far from objective, i. e., preparation- and observation-independent reality, which would have to explain also the experimenter and his choice of the cut.

The relative-reality-of-unitarily-evolving-state (RRUES) approach of this article is close to that of Dieter Zeh [32] (to mention only one of whom I am aware). I have the impression that the difference is mostly in emphasis.

D) According to Zeh "erasure" is a misnomer [33]. Every name given to a phenomenon must disregard most of the aspects of the latter, but it should catch its main feature. Hence, perhaps "revival" or "recoherence" 32] (p. 10 ) would be more appropriate. But the term "erasure" made history, and 
the catchy ring to it has a great part of the merit for this.

E) It is important to point out that in any correlated state vector $|\Phi\rangle_{I, I I}$ one can have disentanglement: measuring, e. g., any complete observable for subsystem $I$ that has nontrivial (distant) effect on the state $\rho_{I I}$ of subsystem $I I$. One thus achieves a decomposition of $\rho_{I I}$. It is distant state decomposition. A decomposition of $\rho_{I I}$ can be understood as a measurement if and only if it is an orthogonal decomposition, when we have the special case of distant measurement [8].

Disentanglement produces an actually decomposed state: the entanglement in $|\Phi\rangle_{I, I I}$ is eliminated. Naturally, this is true in the following relative sense: the cut is so chosen that the apparatus that performs the direct measurement on subsystem $I$ is part of the 'subject'. Contrariwise, if the apparatus is part of the 'object', and if its initial state is pure, the entanglement is only elevated to the larger system. (Also this is an example how a mixture can be proper or improper [34] depending on the choice of the cut: in the former choice one deals with actual decomposition, i. e., with a proper mixture; in the latter with an improper one.)

It should be realized that every correlated state vector $|\Phi\rangle_{I, I I}$ allows two mutually incompatible distant state decompositions of $\rho_{I I}$. They are performable, e. g., by measuring two distinct complete observables for subsystem $I$ that have nontrivial distinct (distant) effects on $\rho_{I I}$. By definition, two state decompositions are incompatible if they do not allow a common further decomposition of both. If not both distant state decompositions are orthogonal, i. e., measurements, one does not have EPR-type disentanglement. But physical interpretation may be in conflict with quasiclassical local thinking also in this more general case .

F) It was, of course, quite clear to Scully et al. that simple erasure is an EPR-type experiment ([20], Appendix 2.) It is shown in this article that so is delayed-choice erasure. In the second part of this study, also the random delayed-choice real experiment of Kim et al. [19] is shown to be of this kind.

Englert, Scully and Walther call the EPR paradox [16], addressed at the Copenhagen claim of completeness of quantum-mechanical description, only "EPR problem". They also give a solution to this problem. It is well illustrated by the last sentence in their paper [20]: "... the property of being a first-slit atom (particle $I I$ in the state $|1\rangle_{I I}$, F. H.) is not possessed by 
the atom until the photon is found in the first resonator (until particle $I$ is found in the state $|1\rangle_{I}$, F. H.)".

The RRUES approach of this study suggests a similar solution. Actually, there is not even an EPR problem. The above "property ... possessed" by particle $I I$ is relative to particle $I$ being in the state $|1\rangle_{I}$, or relative to the which-way detector on which the result 'way 1' is displayed. Thus, the seemingly local (or subsystem) property is actually global, and hence there is no problem.

Let me try to explain this in case of the original position and linear momentum EPR-type disentanglement [16]. The bipartite state vector suggested by the authors contains precise correlation both between the positions of the two particles and between their linear momenta. (Let us pretend, for the sake of argument, that it is a normalized, i. e., a correct state vector.) These two correlations are compatible, and hence both objectively present. But the very positions and linear momenta of the particles are only possibilities; not actualities (in the usual sense of these terms).

Considering collapse as a mental process (cf second half of remark C) implies attributing reality to the (coherent) possibilities and the correlations (established in interaction of subsystems). 'Actuality' is the product of the mentioned mental process: when we choose the cut, then in the objectsubject relation we can highlight part of the potentialities elevating them to 'actualities'.

Returning to the original EPR case, this means the following. We can take a definite position of the first particle in the 'subject' (of quantummechanical description), then the second particle has a definite position (in the 'object' of description). Instead, we can measure the position of the first particle, and put the measurement result in the 'subject'. Then the positions of both particles have definite values. Analogously, we can do this for linear momentum. What the quantum-mechanical formalism does not allow us to do is highlighting both position and momentum at the same time. Nevertheless, at the potential level they are both real.

This calls to mind Mermin's famous mantra (in his Ithaca interpretation) "The correlations, not the correlata" 35]. I think this is quantummechanically quite true. (Seevinck's result [36], contrary to the claim of the author, does not seem to cast a shadow on the reality of quantum correlations, which are experimental facts; instead, it seems to show that they can be global.)

The well-known original EPR argument [16] assumed collapse and values 
obtained in measurement as a reality. Hence the fallacy of their conclusion that quantum mechanics can not be considered a complete description of an experiment. In relative-state interpretation it can.

The illusion of the EPR paradox will be discussed again in connection with the real random delayed-choice erasure experiment of Kim et al. [19] near the end of the second part of this study.

G) It has turned out that application of quantum mechanics to macroscopic systems has interesting consequences. I have in mind the EPR-type entanglement described in section 4, which allows serious distant manipulation of the quantum state of the macroscopic body. It appears as if the state of the latter had kind of a classical 'surface', which is stable, robust, and independent of influence from the environment, and a quantum 'interior', which can be distantly manipulated on account of quantum correlations.

H) There was an interesting debate in the American Journal of Physics between Mohrhoff on the one hand and Englert, Scully and Walther on the other. In [37] Mohrhoff challenged the idea of delayed-choice erasure, in particular, its micromaser realization championed by the latter authors [3], 38].

Taking the reality-of-states point of view, as done also in the present article, he pointed out that the EPR-type correlation existing between subsytems $I$ and $I I$ in simple erasure (cf (6a) and (9)) disappears in the act of detection of subsystem II (cf the analogous remark in the passage beneath relation (17) above).

The mentioned defenders of delayed-choice erasure refuted Mohrhoff's argument in two articles [7], [20]. They took the point of view that quantum mechanics is no more than a book-keeping device for calculating probabilities of future experiments. Then, as it was already mentioned, they showed that the relevant probabilities are the same in simple and delayed-choice erasures.

In a subsequent article, Mohrhoff conceded his "error" [39]. It is not surprising that Mohrhoff readily gave up the reality-of-states approach to quantum mechanics if we remember his Pondicherry interpretation [40] (cf Section V there, cf also [41]).

I) To comment on the apparently play-safe attitude of Englert, Scully and Walther, I first quote their words [20] (Concluding remarks there): 
"The state vector ... serves the sole purpose of summarizing concisely our knowledge about the entangled atom-and-photon system..."

One cannot disagree with this unless by "knowledge" they mean only probabilities. That the latter is true is suggested also by the fact that they themselves characterize their attitude as a "minimalistic interpretation of state vectors ... common to all interpretations..." (ibid. p. 328, left column).

On the other hand, it seems to me that the authors surpass the minimalistic position when they say (ibid. p.327, right column) "...this unacceptable view (they mean that of Mohrhoff, F. H.) results from a lack of appreciation of the objective nature of the EPR-type correlations that link photon states to corresponding atom states" (remember that photons are particles $I$ and atoms are particles II in their micromaser case; italics by F. H.).

Correlations are part of the composite-system state; actually, the former determine the latter [35]. If they have an objective nature, it is hard to imagine how the state is deprived of it.

In support to their 'minimalistic' point of view, the authors remind of van Kampen's Theorem 4 [42] where he says:

"Whoever endows the state vector with more meaning than is needed for computing observable phenomena is responsible for the consequences." (Italics by F. H.)

Perhaps one should take responsibility and adopt the reality-of-states attitude, as it is done in this article, and thus, besides being able to compute probabilities, also gain a fuller insight in the quantum-mechanical 'mechanism' of numerous beautiful thought and real experiments. Among them stand out the ones given to us by Scully et al. (cf also Ref-s [5] and [6]).

\section{Appendix A. On the 'partner state' in a canonical Schmidt decom- position}

It was shown in previous work that every bipartite state vector implies an antiunitary, so-called correlation operator $U_{a}$ that maps the range of the first-subsystem reduced density operator onto the (always equally dimensional) range of the second reduced density operator (see a short form in subsection 2.1 in [13] or more details in [8]). The correlation operator maps 
the first state vector onto the second (partner) one in each term of every canonical Schmidt decomposition of the bipartite state vector at issue.

Thus, the correlation operator comes handy in EPR-type entanglement, where there is a non-denumerable infinity of distinct canonical Schmidt decompositions (and $U_{a}$ is one fixed entity). If one such decomposition is given, then one can just read $U_{a}$, and make use of it in any other such decomposition.

In particular, we can in (6a) read that the correlation operator implied by $|\Psi\rangle_{I, I I}$ is determined by the following map of basis onto basis (in an antilinear way):

$$
U_{a}|j\rangle_{I}=|j\rangle_{I I}, \quad j=1,2 .
$$

Then $(8 \mathrm{a}, \mathrm{b})$ is immediately obtained from $(7 \mathrm{a}, \mathrm{b})$.

\section{Appendix B. On simple coherence states}

The requirement of symmetry under exchange of each of the two terms in a canonical Schmidt decomposition of $|\Psi\rangle_{I, I I}$, in view of $(7 \mathrm{a}, \mathrm{b}),(8 \mathrm{a}, \mathrm{b})$ and (9) is equivalent to

$$
e^{i \lambda}=e^{-i \lambda}, \quad e^{i \delta}=e^{-i \delta}, \quad e^{i \gamma}=e^{-i \gamma} .
$$

This is further equivalent to

$$
\lambda=0 \text { or } \pi, \quad \delta=0 \text { or } \pi \quad \gamma=0 \text { or } \pi .
$$

First, we take $\gamma=0$, because it is anyway an open phase factor. Then we are left with 4 possibilities, but $\lambda=0=\delta$ and $\lambda=\pi=\delta$ give coherence vectors distinct by a factor $(-1)$, and so do $\lambda=0, \delta=\pi$ and $\lambda=\pi, \delta=0$. Hence, if we assume for simplicity $p=q$, we are left with the two coherence vectors in (10) as claimed.

The requirement that the exchange operator take one term in a canonical Schmidt decomposition into the other is, in view of $(7 a, b),(8 a, b)$ and $(9)$, again putting $\gamma=0$, equivalent to $p=q, \quad \lambda=0, e^{i(\delta+\pi)}=e^{-i \delta}$, and this is further equivalent to $\delta=\pi / 2$ or $\delta=-\pi / 2$ giving the two coherence vectors of (11) as claimed. 


\section{Appendix C. Illustration of the Role of the Cut in the Relative- State Interpretation. Simple Erasure}

We now take the composite-system state $|\Psi\rangle_{I, I I}$ of (6a) or (12), we introduce both detectors (or set of detectors), $D_{I}$ for particle $I$ and $D_{I I}$ for particle $I I$, as part of the 'object' (of quantum-mechanical description). Let $M$ denote the choice of detector for particle $I: M=w w$ in the which-way measurement, and $M=c o h$ in the coherence measurement. Further, let zero denote the initial, untriggered state of the detectors. At an arbitrary moment $t$, the 'object' is then described by the state vector

$$
\left(U_{D_{I} I}(t) U_{I I D_{I I}}(t)\right)\left(|M, 0\rangle_{D_{I}}|\Psi\rangle_{I, I I}|0\rangle_{D_{I I}}\right) .
$$

Finally, let $d=1,2$ be the possible results of the $M=w w$ measurement, and $d=+,-$ those of the coherence measurement (cf (10)). Then, after the choice of the experimenter whether he wants to have which-way measurement or coherence measurement (erasure), (C.1a) is suitably rewritten in the following two respective versions:

$$
\left(U_{D_{I} I}(t) U_{I I D_{I I}}(t)\right)\left[|M=w w, 0\rangle_{D_{I}}\left((1 / 2)^{1 / 2}\left(|1\rangle_{I}|1\rangle_{I I}+|2\rangle_{I}|2\rangle_{I I}\right)\right)|0\rangle_{D_{I I}}\right] \text {, }
$$

and

$$
\left(U_{D_{I} I}(t) U_{I I D_{I I}}(t)\right)\left[|M=\operatorname{coh}, 0\rangle_{D_{I}}\left((1 / 2)^{1 / 2}\left(|+\rangle_{I}|+\rangle_{I I}+|-\rangle_{I}|-\rangle_{I I}\right)\right)|0\rangle_{D_{I I}}\right]
$$

(we have substituted in (C.1a) (6a) and (12) respectively).

Relations (C.1b) or (C.1c) describe the process as a dynamically closed system, i.e., when the 'object' and 'subject' (or observing system) are dynamically and statistically independent of each other. (Equivalently, any enlargement of the 'object' would include a part of the environment state via a non-interacting tensor product.)

In simple erasure (C.1c) is relevant, and, at an instant $t_{I}$, the detector $D_{I}$ is triggered displaying either that particle $I$ was in the state $|+\rangle_{I}$ or that it was in $|-\rangle_{I}$. The detection of particle $I I$ takes place at a later instant $t_{I I}: t_{I}<t_{I I}$.

In the time interval $t_{I}<t<t_{I I} \quad$ (C.1c) becomes

$$
(1 / 2)^{1 / 2}\left\{\left[U_{D_{I} I}(t)\left(|M=c o h, d=+\rangle_{D_{I}}|+\rangle_{I}\right)\right]\left(U_{I I}(t)|+\rangle_{I I}\right)\left(U_{D_{I I}}|0\rangle_{D_{I I}}\right)+\right.
$$




$$
\left.\left[U_{D_{I} I}(t)\left(|M=\operatorname{coh}, d=-\rangle_{D_{I}}|-\rangle_{I}\right)\right]\left(U_{I I}(t)|-\rangle_{I I}\right)\left(U_{D_{I I}}|0\rangle_{D_{I I}}\right)\right\} .
$$

(Note that the state of photon $I I$ and that of the detector $D_{I I}$ evolve independently of each other.)

Contrariwise, the simplest choice of the cut is such that subsystem $D_{I}$ in the state displaying either + or - , particle $I$ and subsystem $D_{I I}$ are 'moved' to the 'subject'. Then, (C.2) is replaced by $\left(U_{I I}|+\rangle_{I I}\right)$ or by $\left(U_{I I}|-\rangle_{I I}\right)$ respectively. (This is the most common quantum-mechanical 'picture' of simple erasure.)

One can displace the cut half a step back: leave the same subsystems in the 'subject', but without specifying the result of measurement on $D_{I}$. Then instead of (C.2) we have the density operator

$$
\rho_{I I}=(1 / 2)\left(U_{I I}|+\rangle_{I I}\left\langle+\left.\right|_{I I} U_{I I}^{\dagger}+U_{I I} \mid-\right\rangle_{I I}\left\langle-\left.\right|_{I I} U_{I I}^{\dagger}\right) .\right.
$$

Note that also (C.1c) implies $\rho_{I I}$ of (C.3), but as an improper mixed state (cf [34]). In case of the displaced cut defining (C.3), $\rho_{I I}$ is a proper mixture, expressing the lack of knowledge on part of the 'subject' what the result of the measurement by $D_{I}$ is. 


\section{Appendix D. The Equality of Probabilities in Simple and Delayed- choice Erasures}

\section{Simple erasure}

Detector $D_{I}$ detects one of the four possibilities ( $M=w w, d=1,2 ; \quad M=$ coh, $d= \pm$ ) for particle $I$ (see the preceding Appendix for more details). Detector $D_{I I}$ is that of localization of particle $I I$. One can imagine that $D_{I I}$ consists of a number, say $N$, detectors $\left\{D_{I I}^{n}: n=1,2 \ldots, N\right\}$ placed along the vertical $x$ axis. Let us denote by $|d=1\rangle_{I I}$ or $|d=2\rangle_{I I}$ the state vector of particle $I I$ if one has distant which-way measurement, and we denote it by $|d=+\rangle_{I I}$ or $|d=-\rangle_{I I}$ if the distant measurement is of the coherence type. Then immediately before detection, occurring at $t_{I I}$, we have the decomposition

$$
\left|d, t_{I I}-\epsilon\right\rangle_{I I}=\sum_{n=1}^{N} \int_{x_{n}-(\Delta x) / 2}^{x_{n}+(\Delta x) / 2}\left(\left\langle\left. x\right|_{I I} \mid d, t_{I I}-\epsilon\right\rangle_{I I}\right)|x\rangle_{I I} d x, \quad 0<\epsilon \ll 1,
$$

where $x_{n}$ is at the center of the $n$-th detector, $\Delta x$ is the width of the detectors, and $N \Delta x$ is a large enough span so that the photon is with certainty detected (in one of the $N$ detectors):

$$
\left|\int_{x_{1}-(\Delta x) / 2}^{x_{N}+(\Delta x) / 2}\left(\left\langle\left. x\right|_{I I} \mid d, t_{I I}-\epsilon\right\rangle_{I I}\right) d x\right|^{2}=1 .
$$

State-vector decomposition (D.1a) can be viewed as a coherent set of $N$ possibilities (expressed by the $N$ component terms in (D.1a)), of which precisely one will be realized in the localization measurement for the individual particle $I I$. The probability $p_{n}(d)$ of the $n$-th possibility to be realized is, as it is well known,

$$
\left.p_{n}(d)=\lim _{\epsilon \rightarrow 0} \mid \int_{x_{n}-(\Delta x) / 2}^{x_{n}+(\Delta x) / 2}\left\langle\left. x\right|_{I I} \mid d, t_{I I}-\epsilon\right\rangle_{I I}\right)\left.d x\right|^{2}
$$

because coherence implies adding (this time via integrals) the amplitudes and not the probabilities.

Note that the certain detection in $D_{I I}$ expressed by (D.1b) has to be replaced by $\sum_{n=1}^{N} p_{n}(d)=1$ in spite of the coherence in (D.1a). Namely, when the measurement is performed, it deletes the coherence expressed by the sum in (D.1a). 
Since $\left|d, t_{I I}-\epsilon\right\rangle_{I I}=U_{I I}\left(t_{I I}-\epsilon\right)|d\rangle_{I I}$, substitution in (C.2) makes it clear that the probability of the result $d= \pm$ of distant coherence measurement and of localization in detector $D_{n}$ for simple erasure is

$$
p_{\text {coh }}^{\text {simple }}(d, n)=(1 / 2) p_{n}(d) .
$$

In case of which-way distant measurement, the probability formula is analogous.

\section{Delayed-choice erasure}

This erasure is characterized by $M=c o h, d= \pm$, and $t_{I I}<t_{I}$, i. e., localization of particle $I I$ is detected earlier than the detection of particle $I$ takes place.

The state vector (C.1c) then becomes in the time interval $t_{I I}<t \leq t_{I}$ :

$$
\begin{gathered}
(1 / 2)^{1 / 2}\left\{\left[\left(U_{D_{I}}(t)|M=\operatorname{coh}, 0\rangle_{D_{I}}\right)\left(U_{I}(t)|+\rangle_{I}\right)\right] \otimes\left[U_{I I D_{I I}}(t)\left(|+\rangle_{I I}|0\rangle_{D_{I I}}\right)\right]+\right. \\
\left.\left[\left(U_{D_{I}}(t)|M=\operatorname{coh}, 0\rangle_{D_{I}}\right)\left(U_{I}(t)|-\rangle_{I}\right)\right] \otimes\left[U_{I I D_{I I}}(t)\left(|-\rangle_{I I}|0\rangle_{D_{I I}}\right)\right]\right\} \cdot(D .4)
\end{gathered}
$$

(Note that this time the state of detector $D_{I}$ and that of photon $I$ evolve independently of each other.)

It is suitable to replace $t$ by $t^{\prime} \equiv t-t_{I I}$, i. e., to consider the time from the detection of particle II (and not from leaving the slits). For simplicity, we write again $t$ instead of $t^{\prime}$.

Taking the limiting form of relation (D.1a) for $\epsilon \rightarrow 0$, for the relevant results $d= \pm$, and omitting $t=0$, one has

$$
|d= \pm\rangle_{I I}=\sum_{n=1}^{N} \int_{x_{n}-(\Delta x) / 2}^{x_{n}+(\Delta x) / 2}\left(\left\langle\left. x\right|_{I I} \mid d= \pm\right\rangle_{I I}\right)|x\rangle_{I I} d x .
$$

Substituting this in (D.4), one obtains

$$
\begin{gathered}
(1 / 2)^{1 / 2} \sum_{n=1}^{N} \sum_{d= \pm}\left\{\left[\left(U_{D_{I}}(t)|M=\operatorname{coh}, 0\rangle_{D_{I}}\right)\left(U_{I}(t)|d= \pm\rangle_{I}\right] \otimes\right.\right. \\
\left.\left[U_{I I D_{I I}}(t)\left(\int_{x_{n}-(\Delta x) / 2}^{x_{n}+(\Delta x) / 2}\left(\left\langle\left. x\right|_{I I} \mid d= \pm\right\rangle_{I I}\right)|x\rangle_{I I} d x|0\rangle_{D_{I I}}\right)\right]\right\} .
\end{gathered}
$$


Now we can ask the question what is the probability $p_{c o h}^{\text {del-ch. }}(d, n)$ of choosing to do coherence distant measurement, i. e., of having $d= \pm$ for particle $I I$, and of its detection in the detector $D_{I I}^{n}$ at $t_{I I}$. The composite-system state vector in (D.5) is suitably decomposed, and we can read the answer. It is

$$
\left.p_{\text {coh }}^{\text {del. }- \text { ch. }}(d, n)=(1 / 2)\left|\int_{x_{n}-(\Delta x) / 2}^{x_{n}+(\Delta x) / 2}\left(\left\langle\left. x\right|_{I I} \mid d= \pm\right\rangle_{I I}\right)\right| x\right\rangle\left._{I I} d x\right|^{2} .
$$

In view of (D.3) and (D.2), (D.6) implies the claimed result

$$
p_{\text {coh }}^{\text {del. }- \text { ch. }}(d= \pm, n)=p_{\text {coh }}^{\text {simple }}(d= \pm, n) \quad n=1,2, \ldots, N .
$$

\section{References}

[1] Jaynes, E. T.: in Foundation of Radiation Theory and Quantum Electronics, Barut A. ed., Plenum, 1980; also item 38 of the Jaynes bibliography on the web

[2] Scully, M. O., Drühl, K.: Quantum eraser: A proposed photon correlation experiment concerning observation and "delayed-choice" in quantum mechanics. Phys. Rev. A 25, 2208-2213 (1982)

[3] Scully, M. O., Englert B.-G., Walther H.: Quantum optical tests of complementarity. Nature 351, 111-116 (1991)

[4] Wheeler J. A.: The 'past' and the delayed-choice double-slit experiment. In: Marlow A. R. ed. Mathematical Foundations of Quantum Theory. Academic Press, New York (1978), pp. 9-48

[5] Kwiat P. G., Steinberg A. M., Chiao R. Y.: Observation of a "Quantum Eraser": A Revival of Coherence in a Two-photon Interference Experiment. Phys. Rev. A 45, 77297739 (1992)

[6] Herbut F., Vujičić M.: First-quantisation quantum-mechanical insight into the Hong-Ou-Mandel two-photon interferometer with polarizers and its role as a quantum eraser. Phys. Rev. A 56, 1-5 (1997) 
[7] Scully M. O., Walther H.: An operational analysis of quantum eraser and delayed choice. Found. Phys. 28, 399-413 (1998)

[8] Herbut F., Vujičić M.: Distant measurement. Ann. Phys (N. Y.) 96, 382-405 (1976)

[9] Schrödinger E.: Discussion of probability relations between separated systems. Proc. Cambridge Phil. Soc. 31, 555-563 (1935)

[10] T. Young, Trans. R. Soc. XCII 12, 387 (1802)

A quantum-mechanical discussion in [43]

[11] Feynman R. P., Leighton R. B., Sands M.: The Feynman Lectures on Physics. Vol. III, Addison Wesley, Reading, (1965), pp. 1-9

[12] Joos E., Zeh H. D.: The emergence of classical properties through interaction with the environment. Zeitschr. Phys. B 59, 223-243 (1985)

[13] Herbut F.: Quantum probability law from 'environment-assisted invariance' in terms of pure-state twin unitaries. J. Phys. A: Math. Theor. 40, 5949-5971 (2007); also arXiv: quant-ph/ 0611220

[14] Vujičić M., Herbut F.: A quantum-mechanical theory of distant correlations. J. Math. Phys. 25, 2253-2259 (1984)

[15] Cantrell C. D., Scully M. O.: The EPR paradox revisited. Phys. Rep. 43, 499-508 (1978)

[16] Einstein A., Podolsky B., Rosen N.: Can quantum-mechanical description of physical reality be considered complete? Phys. Rev. 47, 777-780 (1935)

[17] Aspect A., Grangier P.: Experiments on Einstein-Podolsky-Rosen-type correlations with pairs of visible photons. In Proc. Int. Symp. Foundations of Quantum Mechanics. Tokyo (1983), pp.214-224

[18] Bennett C. H., Brassard G., Crépeau C., Jozsa R., Peres A., Wootters W. K.: Teleporting an unknown quantum state via dual classical and Einstein-Podolsky-Rosen channels. Phys. Rev. Lett. 70, 1895-1899 (1993) 
[19] Kim Y.-H., Yu R., Kulik S. P., Shih Y., Scully M. O.: Delayed "choice" quantum eraser. Phys. Rev. Lett. 84, 1-5 (2000); also arXiv: quant-ph/9903047

[20] Englert B.-G., Scully M. O., Walther H.: Quantum erasure in doubleslit interferometers with which-way detectors. Am. J. Phys. 67, 325-329 (1999)

[21] Bohr N.: Atomic Physics and Human Knowledge. Science Editions Inc., New York (1961)

[22] Shimony A.: Role of the observer in quantum theory. Am. J. Phys. 31, 755-773 (1963)

[23] Friedman J. R., Patel V., Chen W., Tolpygo S. K., Lukens J. E.: Quantum superposition of distinct macroscopic states. Nature (London) 406 , 43-46 (2000)

[24] Giulini D., Joos E, Kiefer C., Kupsch J., Stamatescu I.-O., abd Zeh H. D.: Decoherence and Appearance of a Classical World in Quantum Theory, Springer, Berlin, 1996

[25] Zurek W. H.: Decoherence, einselection, and the quantum origins of the classical, Rev. Mod. Phys. 75, 715-775 (2003)

[26] Schlosshauer M.: Decoherence, the measurement problem, and interpretations of quantum mechanics, Rev. Mod. Phys. 76, 1267-1305 (2004); also arXiv: quant-ph/0312059

[27] Von Neumann J.: Mathematical Foundations of Quantum Mechanics. Princeton University Press, Princeton (1955)

[28] Stapp H. P.: The Copenhagen interpretation. Am. J. Phys. 40, 10981116 (1972)

[29] Everett III H.:" Relative state" formulation of quantum mechanics. Rev. Mod. Phys. 29, 454-465 (1957)

[30] Herbut F.: Mott's cloud-chamber theory made explicit and the relativecollapse interpretation of quantum mechanics thus obtained. Int. J. Theor. Phys. 34, 679-700 (1995) 
[31] Rovelli C.: Relational quantum mechanics. Int. J. Theor. Phys. 35, 1637-1678 (1996); also arXiv: quant-ph/9609002

[32] Zeh H. D.: in Science and Ultimate Reality, Barrow J. D., Davies P. C. W., Harper C. J. (Eds.), Cambridge UP, 2004; also arXiv: quant-ph/0204088

[33] Zeh H. D.: "Quantum teleportation" and other quantum misnomers, web essay http://www.rzuser.uni-heidelberg.de/ as3/Teleport.html

[34] D'Espagnat B.: Conceptual Foundations of Quantum Mechanics. Second Edition. W. A. Benjamin, Inc., Reading, Massachusetts (1976), subsection 7.2

[35] Mermin N. D.: The Ithaca interpretation of quantum mechanics. Pramana 51, 549-565 (1998); also arXiv: quant-ph/ 9609013

[36] Seevinck M.: The quantum world is not built up from correlations. Found. Phys. 36, 1573-1586 (2006); also arXiv: quant-ph/0508175v3

[37] Mohrhoff U.: Restoration of interference and the fallacy of delayed choice: Concerning an experiment proposed by Englert, Scully, and Walther. Am. J. Phys. 64, 1468-1475 (1996)

[38] Englert B.-G., Scully M. O., Walther H.: The duality in matter and light. Sci. Am. 271 86-92 (1994)

[39] Mohrhoff U.: Objectivity, retrocausation, and the experiment of Englert, Scully, and Walther. Am. J. Phys. 67, 330-335 (1999)

[40] Mohrhoff U.: What quantum mechanics is trying to tell us. Am. J. Phys. 68, 728-745 (2000); also arXiv: quant-ph/9903051

[41] Mohrhoff U.: The Pondicherry interpretation of quantum mechanics. Pramana 64, 171-185 (2005); also arXiv: quant-ph/0412182

[42] Van Kampen N. G.: Ten theorems about quantum mechanical measurements. Physica A 153, 97-113 (1988)

[43] Herbut F.: Quantum interference viewed in the framework of probability theory. Am. J. Phys. 60, 146-150 (1992) 